\title{
Two-Dimensional Transthoracic Echocardiographic Demonstration of Reduction in Fibrin Content in Purulent Pericarditis Following Intrapericardial Fibrinolytic Agent Administration
}

\author{
Mahmoud Abdelsalam ${ }^{1}$, Cyril Nathaniel ${ }^{1}$, Zeyad Elmarzouky ${ }^{2}$, Subash Dulal ${ }^{2}$, Usama \\ $\mathrm{Habib}^{1}$, Maram Ahmed ${ }^{3}$, Amir Ashiq ${ }^{1}$, and Navin Nanda ${ }^{2}$ \\ ${ }^{1}$ Conemaugh Health System \\ ${ }^{2}$ University of Alabama at Birmingham \\ ${ }^{3}$ Ain Shams University
}

October 25, 2021

\begin{abstract}
We describe an adult patient who presented with purulent pericarditis in whom two-dimensional transthoracic echocardiography demonstrated a marked decrease in the area of the right ventricular wall together with the overlying fibrin following intrapericardial administration of a fibrinolytic agent. Documentation of this decrease by measurements performed and illustrated on two-dimensional images have not been reported previously in an adult patient with purulent pericarditis, to the best of our knowledge.
\end{abstract}

\section{Introduction}

Purulent pericarditis (PP) is a devastating condition with a high mortality rate of around $40 \%$ even after appropriate medical and surgical treatment.[1,2] Surgical pericardiectomy can prevent progression to the feared long-term complication of constrictive pericarditis but carries high mortality of nearly $8 \%$. [3] Intrapericardial administration of a fibrinolytic agent (IFA) such as urokinase has been shown to be effective in preventing constriction in one randomized clinical trial.[4] A decrease in fibrin content which is commonly associated with infection with a consequent decrease in pericardial fibrosis is the suggested mechanism for a decrease in the incidence of pericardial constriction following IFA.[5, 6] Because of this, IFA has been recommended by some in the management of PP. [7, 8] In this report, we present an adult patient with PP in whom two-dimensional transthoracic echocardiography (2DTTE) demonstrated a marked decrease in the area of the right ventricular (RV) wall together with the overlying fibrin following IFA as compared to the baseline. These 2DTTE findings are consistent with a substantial decrease in fibrin content following IFA. To the best of our knowledge, these findings have not been documented before with illustrations of both pre and post IFA 2DTTE measurements in an adult with PP.

\section{Case Description}

A 56-year-old white male with a previous medical history of stroke, hyperlipidemia, hypertension, sacral decubitus ulcer and a long-term indwelling Foley catheter presented with severe dyspnea. His vital status was remarkable for a heart rate of 108 beats per minute, respiratory rate of 22 , blood pressure of $106 / 81 \mathrm{mmHg}$ and temperature of $37^{\circ} \mathrm{C}$. Physical exam was negative for murmurs, rub or gallop. Initial workup was significant for elevated white blood cells at $36.6\left(10^{\times 3} / \mathrm{uL}\right)$, elevated troponin level at $0.45 \mathrm{ng} / \mathrm{L}$ and positive 
urine and blood cultures for methicillin-resistant staphylococcus aureus (MRSA). The ECG showed sinus tachycardia and diffuse ST-elevations in leads I, II, III and V2-V6 with PR-elevation in lead aVR suggestive of pericarditis. To rule out myocardial infarction, coronary angiography was done and showed no significant coronary stenosis. 2DTTE demonstrated a large circumferential pericardial effusion measuring $23 \mathrm{~mm}$ in maximum depth with echogenic material consistent with fibrin overlying the cardiac walls.[9,10] Signs of cardiac tamponade including pulsus paradoxus, plethoric inferior vena cava, RV diastolic collapse and right atrial inversion were present. Both left and right ventricular size and function were normal with no valve abnormalities. Subcostal pericardiocentesis was performed and $490 \mathrm{~mL}$ of cloudy purulent fluid was drained with relief of dyspnea. The culture of pericardial fluid came back positive for MRSA. The patient was begun on daptomycin and piperacillin/tazobactam. A repeat 2DTTE 2 days post pericardiocentesis showed only a small amount of residual effusion, but continued presence of the fibrinous material over the RV wall. Because of this and in an effort to prevent possible progression to constrictive pericarditis, $2 \mathrm{mg}$ of fibrinolytic agent (tissue plasminogen activator, alteplase) diluted in $10 \mathrm{ml}$ of normal saline was administered intrapericardially followed by $10 \mathrm{ml}$ normal saline flush. The pericardial drain was clamped for 24 hours. The next day, the patient drained $450 \mathrm{~mL}$ of pericardial fluid. A repeat 2DTTE 2 days later revealed trivial pericardial effusion (Figures 1A, 1B and 2A, 2B, Movies 1A, 1B and 2A, 2B). In the parasternal long axis view, the area of the RV wall together with fibrin deposition measured by planimetry decreased from $261.9 \mathrm{~mm}^{2}$ to $155.48 \mathrm{~mm}^{2}$, a substantial reduction of $40.6 \%$. In the apical four chamber view, the RV wall plus fibrin area decreased from $270.82 \mathrm{~mm}^{2}$ to $139.4 \mathrm{~mm}^{2}$, also a substantial reduction of $48.5 \%$. Fibrin deposition on the RV wall could be recognized using 2DTTE by its uneven irregular contour/localized bulging on the outer pericardial aspect of the RV wall. However, the inner boundary of fibrin deposit could not be distinguished from the contiguous RV wall and hence planimetry included both the fibrin deposit and RV wall. These declines in RV wall areas in our patient are indicative of marked decrease in the amount of fibrinous material by dissolution following IFA.

Our patient did not suffer from any side effects following IFA such as hemorrhage or cardiac tamponade which have been reported previously.[3, 6, 9] Subsequently, intravenous vancomycin was substituted for daptomycin, and the patient was transferred to an acute care rehabilitation for further treatment and care. After 30 days of inpatient rehabilitation, the patient was discharged in stable condition with no clinical evidence of pericardial constriction.

\section{Discussion and Conclusion}

Demonstration of reduction in fibrin content by pre and post IFA 2DTTE images in PP has been clearly illustrated in pediatric patients.[10-12] However, as far as we can find there is mention in the text but, unlike our patient, there is no documentation with measurements illustrated on pre and post IFA 2DTTE images or movies in any adults presenting with $\mathrm{PP}$ in the literature.[3, 6, 13-23]

In conclusion, we document for the first time to the best of our knowledge, a substantial decrease in fibrin content with measurements shown on 2DTTE images before and following IFA in an adult patient presenting with PP. The decrease in fibrin content following IFA is believed to prevent possible progression to fibrosis with subsequent development of constrictive pericarditis with its high morbidity and mortality in these patients.

\section{Authors contributions:}

Mahmoud Abdelsalam contributed to the acquisition of and interpretation of data, data analysis, writing the article, critical revision of the article, and final approval of the article. Navin Nanda contributed to data analysis, writing the article, critical revision of the article, and final approval of the article. Cyril Nathanial contributed to acquisition of and interpretation of data, data analysis, writing the article, critical revision of the article, and final approval of the article. Zeyad Elmarzouky contributed to data analysis, writing the article, critical revision of the article, and final approval of the article. Subash Dulal contributed to data analysis, writing the article, critical revision of the article, and final approval of the article. Usama Habib contributed to data analysis, writing the article, critical revision of the article, and final approval of the 
article. Maram Ahmed contributed to data analysis, writing the article, critical revision of the article, and final approval of the article. Amir Ashiq contributed to data analysis, writing the article, critical revision of the article, and final approval of the article.

\section{References}

1. Imazio M, Adler Y. Management of pericardial effusion. Eur Heart J. 2013;34:1186-97.

2. Khandaker MH, Espinosa RE, Nishimura RA, et al. Pericardial disease: diagnosis and management. Mayo Clin Proc. 2010;85(6):572-93.

3. Dybowska M, Kazanecka B, Kuca P, et al. Intrapericardial fibrinolysis in purulent pericarditis-case report. Int J Emerg Med. 2015;8:36.

4. Cui HB, Chen XY, Cui CC, et al. Prevention of pericardial constriction by transcatheter intrapericardial fibrinolysis with urokinase. Chin Med Sci J. 2005;20:5-10.

5. Trivedi U, Fell C, Madsen JS, et al. Persistence and progression of staphylococcal infection in the presence of public goods. Npj Biofilms and Microbiomes . 2020;6:55.

6. Dybowska M, Szturmowicz M, Opoka L, et al. Intrapericardial recombinant tissue plasminogen activator in purulent pericarditis- case series. BMC Cardiovasc Disord. 2020;20:392.

7. Wiyeh AB, Ochodo EA, Wiysonge CS, et al. A systematic review of the efficacy and safety of intrapericardial fibrinolysis in patients with pericardial effusion. Int J Cardiol. 2018;250:223-228.

8. Adler Y, Charron P, Imazio M, et al. 2015 ESC Guidelines for the diagnosis and management of pericardial diseases: The Task Force for the Diagnosis and Management of Pericardial Diseases of the European Society of Cardiology (ESC) Endorsed by: The European Association for Cardio-Thoracic Surgery (EACTS). Eur Heart J. 2015;36:2921-2964.

9. Augustin P, Desmard M, Mordant P, et al. Clinical review: intrapericardial fibrinolysis in management of purulent pericarditis. Crit Care. 2011;15:220.

10. Ustünsoy H, Celkan MA, Sivrikoz MC, et al. Intrapericardial fibrinolytic therapy in purulent pericarditis. Eur J Cardiothorac Surg. 2002;22(3):373-6.

11. Juneja R, Kothari SS, Saxena A, et al. Intrapericardial streptokinase in purulent pericarditis. Arch Dis Child . 1999;80(3):275-7.

12. Ideh RC, Pollock L, Sanneh A, et al. Management of persistent purulent pericarditis using streptokinase for intrapericardial fibrinolysis. Paediatrics 83 International Child Health.2014;34(3):220-223.

13. Reznikoff CP, Fish JT, Coursin DB. Pericardial Infusion of Tissue Plasminogen Activator in Fibropurulent Pericarditis. J Intensive Care Med . 2003;18(1):47-51.

14. Schafer M, Lepori M, Delabays A, et al. Intrapericardial urokinase irrigation and systemic corticosteroids: an alternative to pericardectomy for persistent fibrino-purulent pericarditis. Cardiovasc Surg . 2002;10(5):508-11.

15. Winkler WB, Karnik R, Slany J. Treatment of Exudative Fibrinous Pericarditis with Intrapericardial Urokinase. The Lancet (British Edition) . 1994;344(8936):1541-42.

16. Cross JH, De Giovanni JV, Silove ED. Use of streptokinase to aid in drainage of postoperative pericardial effusion. Br Heart J . 1989;62(3):217-219.

17. Mann-Segal DD, Shanahan EA, Jones B, et al. Purulent pericarditis: rediscovery of an old remedy. $J$ Thorac Cardiovasc Surg . 1996;111(2):487-8.

18. Defouilloy C, Meyer G, Slama M, et at. Intrapericardial fibrinolysis: a useful treatment in the management of purulent pericarditis. Intensive Care Med. 1997; 23: 117-118. 
19. Tomkowski WZ, Gralec R, Kuca P, et al. Effectiveness of Intrapericardial Administration of Streptokinase in Purulent Pericarditis. Herz. 2004 ;29:802-805.

20. Abdelnabi M, Almaghraby A, Saleh Y, et al. Acute purulent pericarditis treated conservatively with intrapericardial fibrinolysis and intrapericardial and systemic antibiotics. Proc (Bayl Univ Med Cent) . 2021;34(4):498-499.

21. Bigham MT, Brady PW, Manning PB, et al. Therapeutic application of intrapericardial tissue plasminogen activator in a 4-month-old child with complex fibropurulent pericarditis. Pediatr Crit Care Med. 2008;9(1):e1-e4.

22. Ekim H. Demirbağ R. Intrapericardial Streptokinase for Purulent Pericarditis. Surg Today . 2004;34:569572.

23. Çakir Ö, Gurkan F, Balci AE, et al. Purulent Pericarditis in Childhood: Ten Years of Experience. Journal of Pediatric Surgery . 2002;37(10):1404-08.

Figure and movie legends

Figure 1 A, B. Two-dimensional transthoracic echocardiography. Parasternal long axis view. A. The area of the right ventricular wall together with the overlying fibrin (arrow) measured $261.90 \mathrm{~mm}^{2}$ by planimetry before intrapericardial injection of fibrinolytic agent. B. Following injection, the same wall with fibrin measured $155.48 \mathrm{~mm}^{2}$ in area, a reduction of $40.6 \%$. Movies $1 \mathrm{~A}, 1 \mathrm{~B} . \mathrm{AO}=$ aorta; $\mathrm{LA}=$ left atrium; $\mathrm{LV}=\mathrm{left}$ ventricle; $\mathrm{RA}=$ right atrium; $\mathrm{RV}=$ right ventricle.

Figure 2 A, B. Two-dimensional transthoracic echocardiography. A. Apical four chamber view. The area of the right ventricular wall together with the overlying fibrin (arrow) measured $270.82 \mathrm{~mm}^{2}$ by planimetry before intrapericardial injection of fibrinolytic agent. B. Following injection, the same wall with fibrin measured $139.95 \mathrm{~mm}^{2}$ in area, a reduction of 48.5\%. Movies 2A, 2B. Abbreviations as in Figure 1 and Movies 1A, $1 \mathrm{~B}$.

Supplemental Information. Movies 1A, 1B, 2A, 2B.

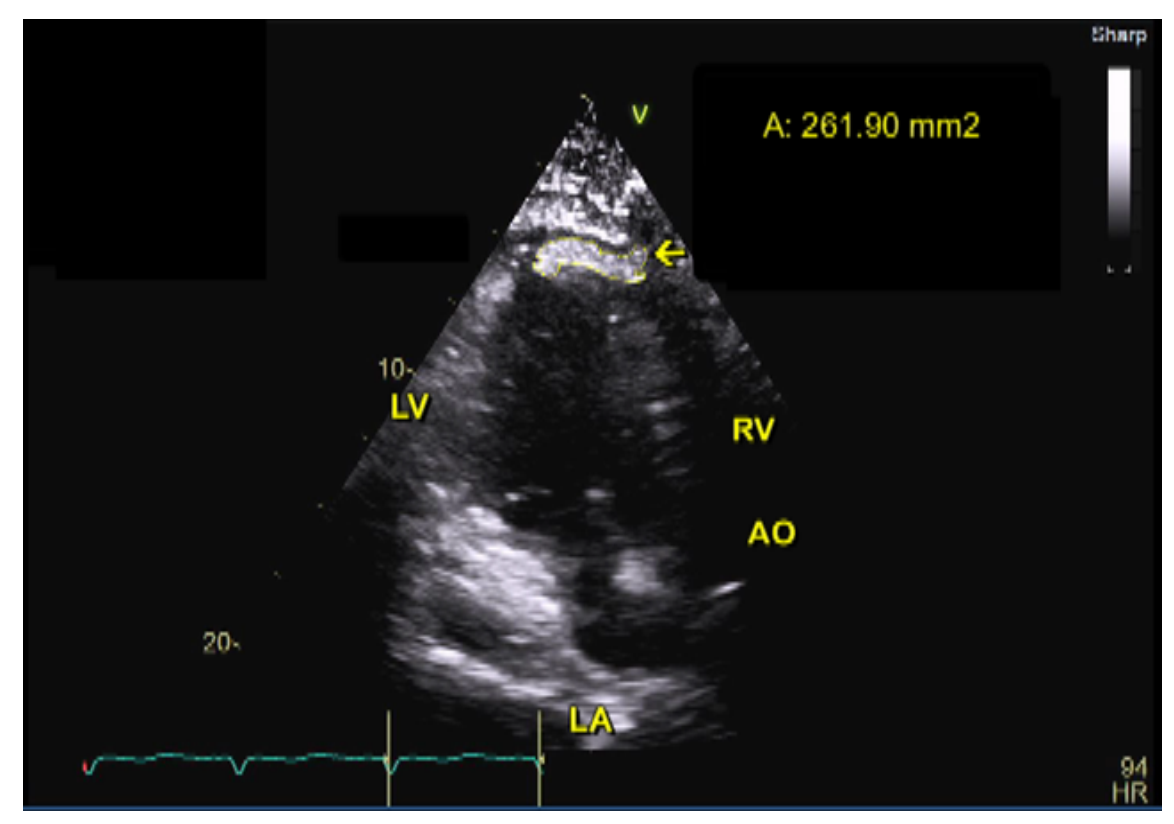



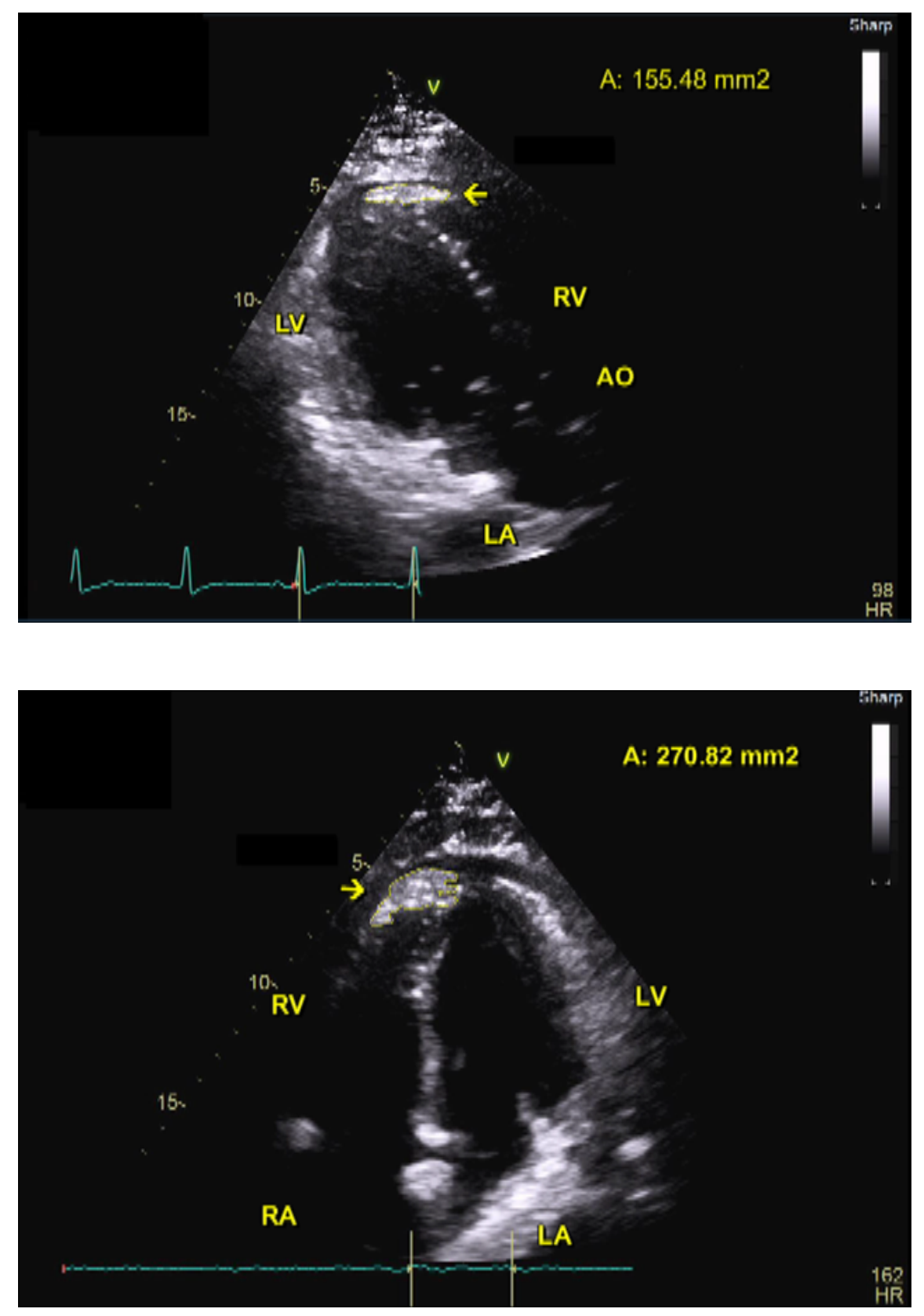


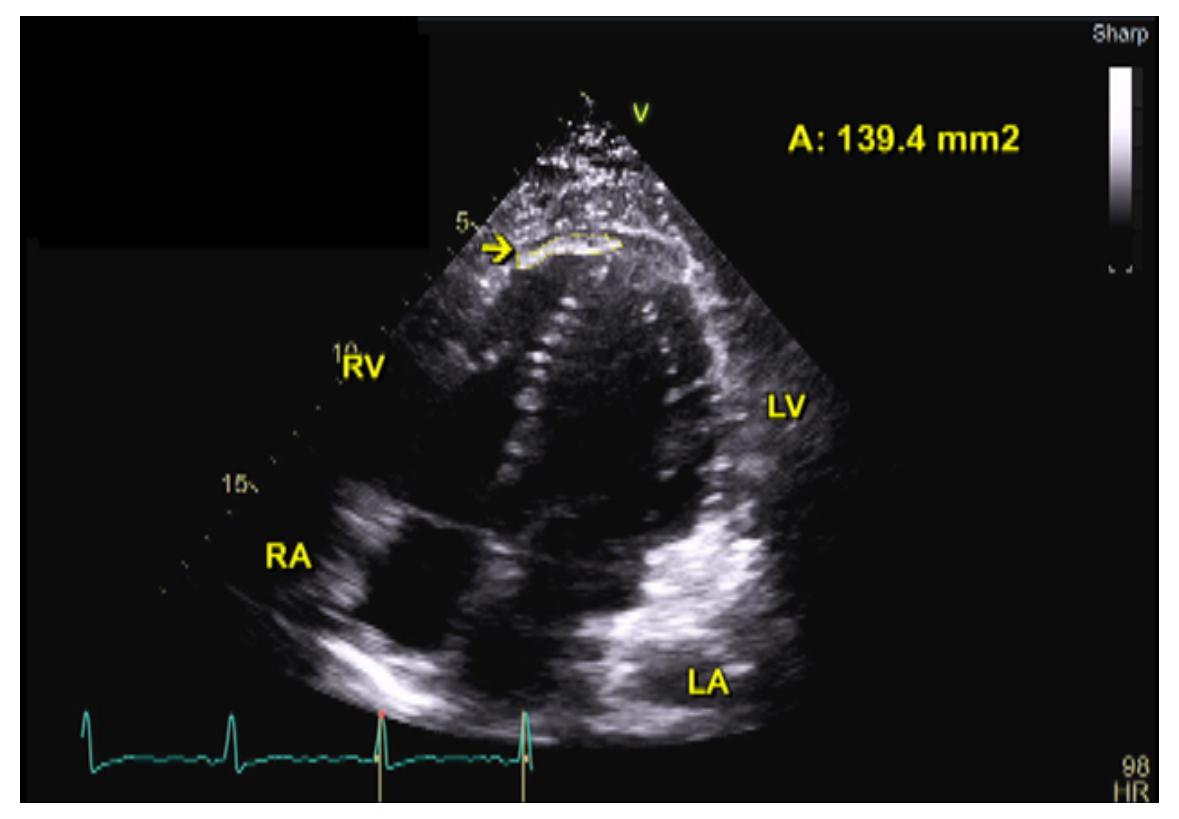

